\title{
Pump-and-Treat : Tecnica di Bonifica a lungo termine in un sito di grandi dimensioni
}

\author{
Federica Russo ${ }^{1}$ \\ ${ }^{1}$ University of Salerno
}

\begin{abstract}
La contaminazione delle acque sotterranee, causata soprattutto da pratiche inadeguate di smaltimento rifiuti, è un problema ormai diffuso e in crescita. L'inquinamento di queste acque spesso si traduce in problemi legati all'approvvigionamento idrico e di conseguenza in rischi significativi per la salute umana. È importante, dunque, prevenire la contaminazione e lì dove non è possibile attuare un'adeguata tecnica di bonifica. Il pump-and-treat $(\mathrm{P} \& \mathrm{~T})$ è una tecnologia convenzionale atta a contenere e controllare pennacchi di contaminanti del sottosuolo e nelle acque sotterranee; l'obiettivo è quello di valutarne l'efficacia attraverso un caso studio nel sud dell'Arizona, i cui risultati sono rappresentati da un set di dati storici corrispondenti a 23 anni di funzionamento di un sistema che inizialmente è solo P\&T e successivamente è accompagnato da altre due tecniche di bonifica .
\end{abstract}

\section{Introduzione al quadro normativo e alla tecnicna di bonifica}

Il degrado qualitativo e quantitativo delle acque sotterranee costituisce una seria problematica ambientale. La forte pressione antropica legata alle attività produttive (scarico incontrollato di reflui di varia origine, apporto di sostanze chimiche per trattamenti antiparassitari e concimazioni,ecc) ha posto una crescente attenzione verso una corretta tutela e gestione delle risorse idriche sotterranee. In Italia, la gestione delle acque sotterranee emunte è regolata dall' Art. 243 della Parte IV del 
D.Lgs. 152/2006. Questa gestione deve essere realizzata secondo i principi di precauzione e riduzione dell'inquinamento e prevede che nel caso in cui vengano superati i valori limite fissati dalla normativa, le acque sotterranee devono essere sottoposte a specifici trattamenti per il ripristino del loro stato naturale.

Tra i numerosi trattamenti di bonifica utilizzati per le acque sotterranee, i sistemi "Pump\&Treat" sono diventati ampliamente utilizzati all'inizio degli anni ' 80 per effetto di una generale semplicità costruttiva e buona efficienza ${ }^{1}$. Con questo metodo, uno o più pozzi di estrazione vengono utilizzati allo scopo di creare una depressione piezometrica per rimuovere l'acqua contaminata dal sottosuo$\mathrm{lo}^{2}$. Tale depressione può avere un duplice effetto, ovvero deviare le linee di flusso, preservando le captazioni poste più a valle, e determinare un abbassamento del livello della falda in modo tale da controllare e interrompere la migrazione a valle del pennacchio di inquinante. Affinché l'intervento sia funzionale è necessario che la depressione creata permetta di captare tutta l'acqua contaminata, mantenendo la velocità di estrazione al valore minimo sufficiente a prevenire l'allargamento della zona contaminata ${ }^{3}$. L'acqua contaminata pompata dal sottosuolo è poi diretta ad un serbatoio e/o inviata ad un impianto di trattamento fuori terra (strippaggio dell'aria, adsorbimento del carbonio, sistema di trattamento biologico oppure processi di tipo chimico per trattamenti di breve durata). Una volta depurata, può essere riversata in fognatura o per migliorare il processo di bonifica può essere reinserita a monte della zona inquinata, in questo modo si ha l'aumento della portata di ricircolazione nella zona da trattare migliorando il recupero dell'inquinante. Un'illustrazione di un trattamento Pump\&Treat con reinserimento in falda è fornita in FIG.1

Gli studi sul funzionamento dei sistemi di P\&T hanno dimostrato che la tecnica ha molto successo nel contenere pennacchi contaminanti e, in alcuni casi, nel ridurli. Tuttavia, il pump-and-treat è generalmente inefficace per rimuovere completamente i contaminanti dal sottosuolo ${ }^{4}$. Esistono 
molti fattori che possono limitare l'efficacia di rimozione dei contaminanti, tra cui:

1. Presenza di zone a bassa permeabilità (ad es. Lenti di limo / argilla)

2. Desorbimento non lineare, a velocità limitata.

3. Presenza di liquido immiscibile

Infatti, la presenza di sostanze contaminanti sulla fase solida e fasi organiche separate dalla fase acquosa (NAPL) può generare fenomeni quali il "tailing e rebound" che vanno a limitare l'efficacia di questa tecnologia. Il "tailing" consiste nella riduzione progressiva della velocità di estrazione delle sostanze inquinanti e ciò ha come conseguenza il fatto che i tempi per raggiungere gli obiettivi di qualità fissati come obiettivi della bonifica siano molto superiori a quelli previsti sulla base delle equazioni di diffusione (analisi del rischio). Il "rebound" si presenta, invece, in quanto, a seguito di una interruzione delle operazioni di estrazione si osserva, alla ripresa di esse, un aumento della concentrazione delle sostanze in soluzione ${ }^{3}$. Al procedere dell'estrazione, la concentrazione può di nuovo diminuire. Questi due fenomeni sono dovuti a più fattori di tipo chimico e fisico riguardanti la natura del contaminante, il tipo di sottosuolo presente e l'acqua di falda stessa ${ }^{3}$. I principali aspetti da tenere in considerazione in fase di progettazione $\mathrm{P} \& \mathrm{~T}$ sono:

- la portata totale di acqua sotterranea che deve essere emunta dal sottosuolo;

- $\quad$ numero, ubicazione e modalità costruttive e di completamento dei pozzi di estrazione;

- $\quad$ il tempo previsto per realizzare la bonifica dell'area;

- $\quad$ il sistema di monitoraggio;

Questi parametri permettono di ricostruire il quadro completo di riferimento per la situazione specifica. 


\section{Caso studio}

Il caso studio su cui è stata posta l'attenzione è stato svolto nel sito federale Superfund di Tucson International Airport Area (TIAA) nel sud dell'Arizona ${ }^{5}$. Il sito TIAA è stato inserito nell'elenco delle priorità nazionali degli Stati Uniti nell'agosto 1983 in risposta al rilevamento del tricloroetene nelle acque sotterranee da diversi pozzi di approvvigionamento di acqua potabile ${ }^{6}$. Il tricloroetene, che fa parte dei composti di solventi organici, è uno dei contaminanti più comuni nelle acque sotterranee a causa del loro precedente uso diffuso per numerose applicazioni industriali e commerciali ${ }^{7}$. Nella parte superiore della falda acquifera regionale, che è la fonte primaria di acqua potabile per l'area metropolitana di Tucson, esiste un grande pennacchio . La falda acquifera regionale è ricoperta da uno strato di argilla, che va da 1,5 a 7,5 m di spessore, che sembra agire come un'unità confinante e si trova tra i 30 ai 40 m sotto la superficie del suolo. La direzione generale del flusso delle acque sotterranee in condizioni naturali è nord-nord-ovest. Il sito di studio si trova nella sezione sud (vedi Fig. 2).

In Fig. 2. sono mostrate le posizioni dei pozzi di estrazione e le tre principali zone di origine, identificate per la sezione sud del complesso TIAA - Siti 2, 3 e 5. Nel 1987 è stato avviato un grande progetto di bonifica delle acque sotterranee attraverso il P\&T e l'ausilio di altre due tecniche di bonifica (Soil Vapor Extraction e Ossidazione chimica in situ) nella sezione sud del sito. I campioni di acque sotterranee estratti sono stati raccolti una o due volte a settimana per 23 anni di funzionamento del sistema, progettato e utilizzato per catturare l'intero pennacchio. Il set di dati utilizzati per questa analisi comprende le concentrazioni per i campioni raccolti poco prima della fase di trattamento, mentre le portate di estrazione sono state monitorate continuamente. I campioni sono stati raccolti utilizzando protocolli standard approvati e analizzati dall' Agenzia per la protezione ambientale degli Stati Uniti (EPA). I valori di portata di massa dei contaminanti sono stati calcolati per ciascun periodo di misurazione come prodotto del pompaggio del pozzo di estrazione e concentrazione media dei contaminanti. Questi valori sono equivalenti all'entità della massa di 
contaminante rimossa per il dato periodo di misurazione. I dati vengono in genere convertiti in unità standard come il chilogrammo al giorno. Si ha così una interessante valutazione della portata di massa di contaminanti per un'operazione di bonifica molto ampia e a lungo termine. In Fig. 3. è mostrato l'andamento della portata di massa in funzione del tempo.

Il progetto di bonifica è iniziato con il solo sistema P\&T ed è stato possibile osservare un valore di picco della portata di massa di circa $7 \mathrm{~kg} /$ giorno, mentre dopo il terzo anno è diminuito ad un valore di circa $2 \mathrm{~kg} /$ giorno per un periodo stazionario. Negli anni 8-9 (vedi fig.3 a) è avvenuto l'avvio delle operazioni di Soil Vapor Extraction (SVE) nelle zone di origine e sono state rimosse circa quattro volte in più le quantità rimosse con il solo P\&T. Ciò ha suggerito che la maggior parte della massa era inizialmente presente nella fase vadosa. Nell' anno 13, invece, al termine dello SVE e sempre nelle zone di origine, è stata avviata l'ossidazione chimica in situ (ISCO) (vedi fig.3 a). La riduzione dei primi tre anni riflette il significativo impatto della zona di sorgente altamente contaminata, a causa delle grandi quantità di solvente smaltite al suo interno ${ }^{8}$. La graduale riduzione della portata di massa totale osservata durante il periodo successivo, invece, può essere attribuita a vincoli, ovvero la massa immagazzinata in un'unità a bassa permeabilità. È stato stimato infatti che le tre zone di origine abbiano contribuito per circa i due terzi della portata totale durante questo periodo, con il sito 3 che ha contribuito per circa la metà (portata di massa di contaminanti di $1 \mathrm{~kg}$ / giorno). Questi risultati sono stati esaminati da ${ }^{6}$ e attraverso dei modelli presentati da ${ }^{9}$ è stato possibile simulare l'andamento della portata di massa con e senza il risanamento della zona di origine. La simulazione, infine, è stata confrontata con i dati reali misurati dall'inizio dell' ISCO e ne hanno fornito una perfetta rappresentazione. Pertanto, secondo questa analisi il comportamento stazionario della fase P\&T sarebbe persistito per un periodo significativo senza l'ausilio di altri interventi di risanamento nella zona di origine (vedi fig.3 b). Ad esempio, affinché il sistema di P\&T raggiunga la portata di massa finale ( 0,2 kg / giorno) si stima che sarebbero occorsi circa 65 anni dall'anno 9 e che l'attuazione degli interventi di risanamento della zona di origine abbia effettivamente eliminato circa 50 anni di operazioni di P\&T. I risultati, ottenuti attraverso la simu- 
lazione, confermano la scelta di una risoluzione di bonifica migliore ottenuta grazie all'intervento di SVE e ISCO.

\section{Conclusioni}

In definitiva, attraverso un'analisi e un set di dati storici ottenuti da 23 anni di funzionamento di un grande sistema di bonifica (inizialmente solo P\&T e successivamente anche SVE ed ISCO) è stato possibile produrre una grande quantità di informazioni. Si può affermare, infatti, che con l'approccio del solo $\mathrm{P} \& \mathrm{~T}$ il contenimento idraulico del pennacchio è possibile, ma bisogna tener conto che presenta problemi di ottimizzazione dal punto di vista tecnico economico. Per cui, la pulizia totale di una falda acquifera contaminata richiede lunghi periodi di trattamento e in alcuni casi anche altri interventi di bonifica con conseguenti costi totali elevati.

\section{References}

1.Langwaldt, J. H. \& Puhakka, J. A. On-site biological remediation of contaminated groundwater: a review. Environmental Pollution 107, 187-197 (2000).

2.Brusseau, M. L. Soil and Groundwater Remediation. in Environmental and Pollution Science 329-354 (Elsevier, 2019). doi:10.1016/b978-0-12-814719-1.00019-7.

3.Nardo, A. D., I.Bortone \& D.Musmarra. Studio del sito contaminato di Masseria del PozzoSchiavi nel comune di Giugliano in Campania - Considerazioni quantitative sull'efficacia della tecnologia di bonifica Pump and Treat. (Abbiabbè edizioni, 2010).

4.Brusseau, M. L. et al.. Source-zone characterization of a chlorinated-solvent contaminated Superfund site in Tucson AZ. Journal of Contaminant Hydrology 90, 21-40 (2007).

5.DD.Graham, TJ.Allen, ML.Barackman, DiGuiseppi, W. H. \& MF.Wallace. Trichloroethylene 
and 1,1-Dichlorothylene Concentrations in Ground Water After Temporary Shutdown of the Reclamation Well Field at Air Force Plant 44, Tucson, Arizona. (U. S. Geol. Survey, Water-Resources Investigation Report 01-4177, 1999).

6.Brusseau, M. L., Hatton, J. \& DiGuiseppi, W. Assessing the impact of source-zone remediation efforts at the contaminant-plume scale through analysis of contaminant mass discharge. Journal of Contaminant Hydrology 126, 130-139 (2011).

7.Guo, Z., Brusseau, M. L. \& Fogg, G. E. Determining the long-term operational performance of pump and treat and the possibility of closure for a large TCE plume. Journal of Hazardous Materials 365, 796-803 (2019).

8.Brusseau, M. L. Use of Historical Pump-and-Treat Data to Enhance Site Characterization and Remediation Performance Assessment. Water Air, \& Soil Pollution 224, (2013).

9.Zhang, Z. \& Brusseau, M. L. Nonideal transport of reactive solutes in heterogeneous porous media: 5. Simulating regional-scale behavior of a trichloroethene plume during pump-and-treat remediation. Water Resources Research 35, 2921-2935 (1999). 


\section{Figure Captions}

Figure 1. Risanamento della contaminazione delle acque sotterranee mediante il processo di P\&T.

Figure 2. Diagramma dell'area di studio, con le posizioni dei pozzi di estrazione utilizzati per il sistema di P\&T (tratto da ${ }^{6}$ )

Figure 3. Portata di massa di contaminanti in funzione del tempo. a) sistema P\&T completo di SVE ed ISCO; b) rappresentazione approssimativa del solo sistema P\&T. Il tempo zero corrisponde all'inizio dell'operazione di P\&T-aprile 1987 


\section{Figures}

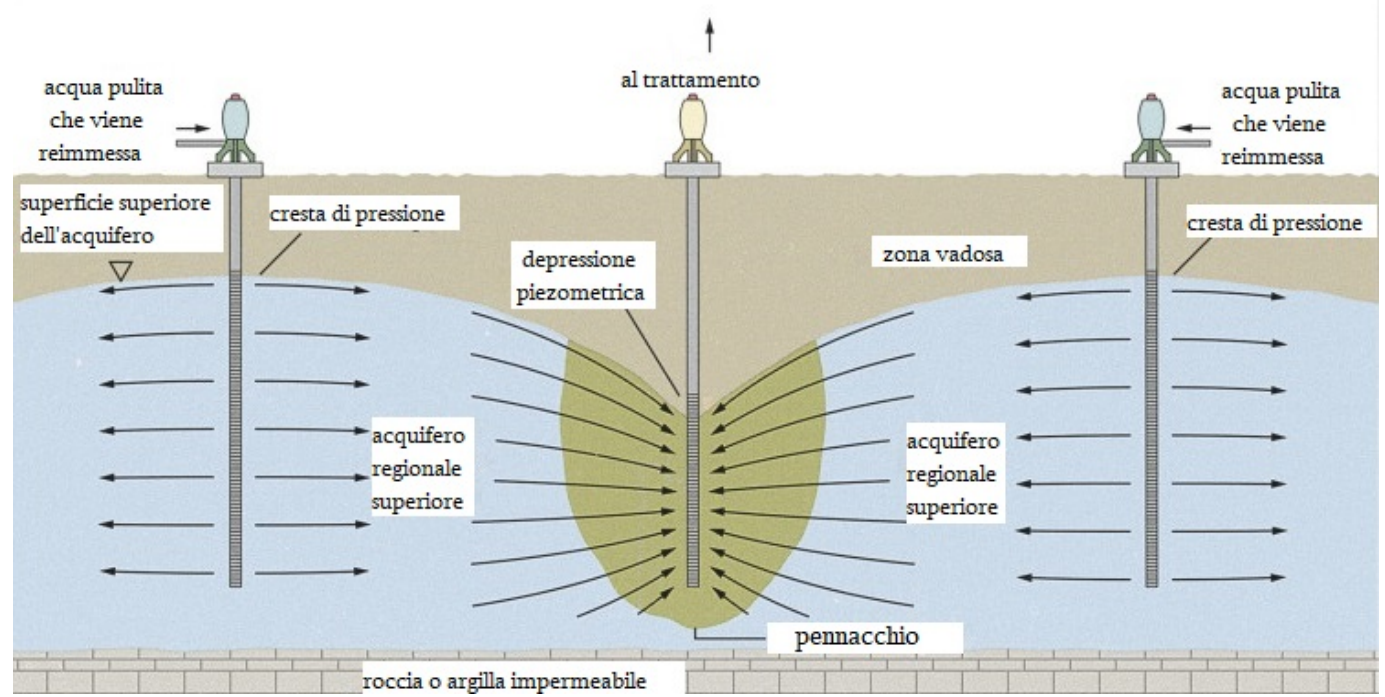

Figure 1: Risanamento della contaminazione delle acque sotterranee mediante il processo di P\&T.

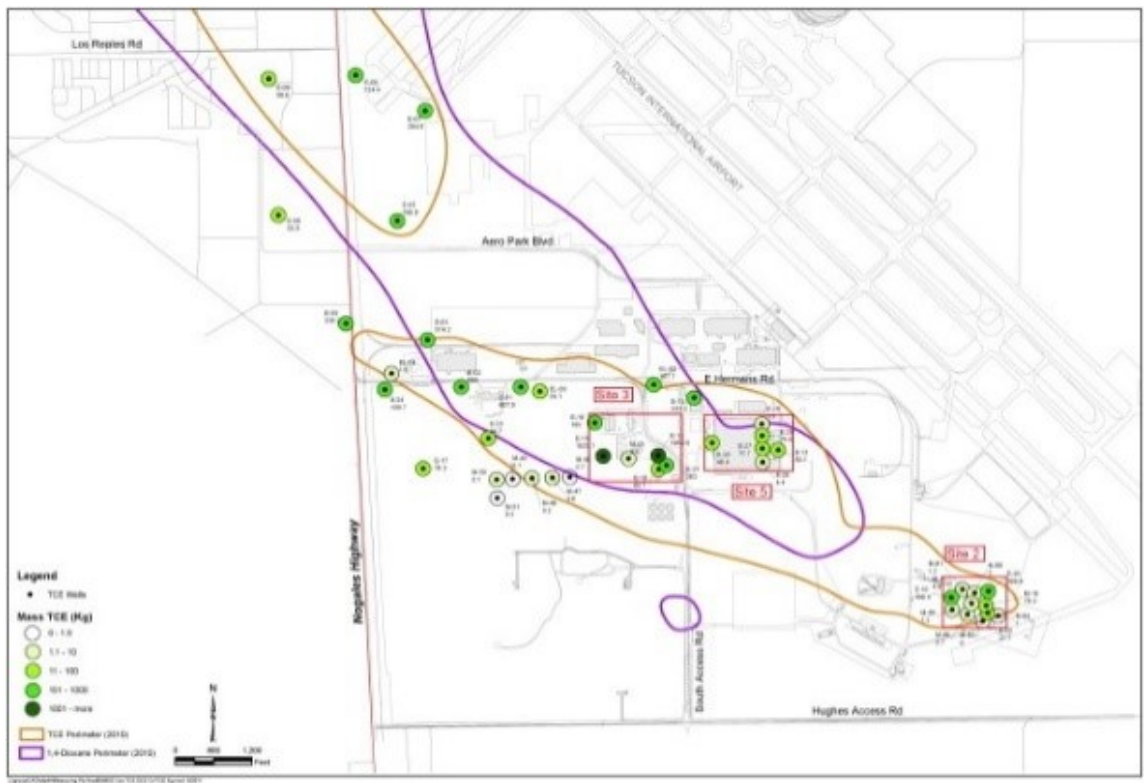

Figure 2: Diagramma dell'area di studio, con le posizioni dei pozzi di estrazione utilizzati per il sistema di P\&T (tratto da ${ }^{6}$ ) 

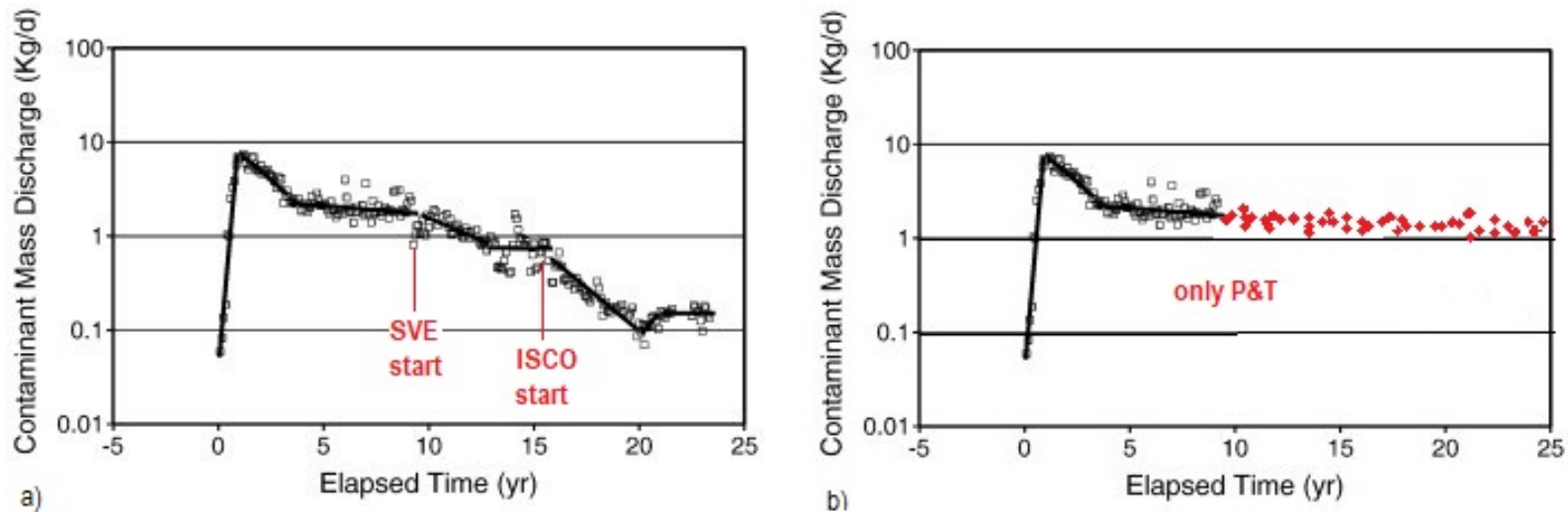

Figure 3: Portata di massa di contaminanti in funzione del tempo. a) sistema P\&T completo di SVE ed ISCO; b) rappresentazione approssimativa del solo sistema P\&T. Il tempo zero corrisponde all'inizio dell'operazione di P\&T-aprile 1987 